\title{
ON A PARAMETRIZATION OF POSITIVE SEMIDEFINITE MATRICES WITH ZEROS
}

\author{
MATHIAS DRTON AND JOSEPHINE YU
}

\begin{abstract}
We study a class of parametrizations of convex cones of positive semidefinite matrices with prescribed zeros. Each such cone corresponds to a graph whose non-edges determine the prescribed zeros. Each parametrization in this class is a polynomial map associated with a simplicial complex supported on cliques of the graph. The images of the maps are convex cones, and the maps can only be surjective onto the cone of zero-constrained positive semidefinite matrices when the associated graph is chordal and the simplicial complex is the clique complex of the graph. Our main result gives a semialgebraic description of the image of the parametrizations for chordless cycles. The work is motivated by the fact that the considered maps correspond to Gaussian statistical models with hidden variables.
\end{abstract}

\section{INTRODUCTION}

For a positive integer $m$, let $[m]=\{1, \ldots, m\}$. Denote the power set of $F \subseteq[m]$ by $2^{F}$. A collection of subsets $\Delta \subseteq 2^{[m]}$ is a simplicial complex if $2^{F} \subseteq \Delta$ for all $F \in \Delta$. The elements of $\Delta$ are called faces and the inclusion-maximal faces are the facets. The ground set of $\Delta$ is the union of its faces. The underlying graph $G(\Delta)$ is the simple undirected graph with the ground set as vertex set and the 2-element faces as edges. All simplicial complexes appearing in this paper are assumed to have ground set $[m]$ and, thus, all underlying graphs have vertex set $[m]$. We make this assumption explicit by speaking of a simplicial complex on $[\mathrm{m}]$.

Let $\mathbb{S}^{m}$ be the $m(m+1) / 2$ dimensional vector space of symmetric $m \times m$ matrices. For an undirected graph $G$ with vertex set $V(G)=[m]$ and edge set $E(G)$, define the $|E(G)|+m$ dimensional subspace

$$
\mathbb{S}^{m}(G)=\left\{\Sigma=\left(\sigma_{i j}\right) \in \mathbb{S}^{m}: \sigma_{i j}=0 \text { if } i \neq j \text { and }\{i, j\} \notin E(G)\right\}
$$

containing the symmetric matrices with zeros at the non-edges of $G$. Let $\mathbb{S}_{\succ 0}^{m} \subset \mathbb{S}^{m}$ be the convex cone of positive semidefinite matrices and $\mathbb{S}_{\succeq 0}^{m}(G)=\mathbb{S}_{\succeq 0}^{m} \cap \mathbb{S}^{m}(G)$ the convex subcone of matrices with zeros prescribed by the graph.

This paper is concerned with particular parametrizations of the graphical cone $\mathbb{S}_{\succeq 0}^{m}(G)$. For a subset $\Delta \subseteq 2^{[m]}$, define the polynomial map

$$
\phi_{\Delta}: \prod_{F \in \Delta} \mathbb{R}^{|F|} \rightarrow \mathbb{S}_{\succeq 0}^{m}
$$

given by

$$
\phi_{\Delta}(\gamma)=\Gamma(\gamma) \Gamma(\gamma)^{T}
$$

Date: October 29, 2018. 
where, for $\gamma=\left(\gamma_{i, F}: F \in \Delta, i \in F\right)$, the $[m] \times \Delta$ matrix $\Gamma(\gamma)$ has entries

$$
\Gamma(\gamma)_{i, F}= \begin{cases}\gamma_{i, F} & \text { if } i \in F \\ 0 & \text { otherwise }\end{cases}
$$

The coordinates of the map are

$$
\phi_{\Delta}(\gamma)_{i j}=\sum_{F \in \Delta: i, j \in F} \gamma_{i, F} \gamma_{j, F}, \quad i, j \in[m] .
$$

In particular, the diagonal coordinates

$$
\phi_{\Delta}(\gamma)_{i i}=\sum_{F \in \Delta: i \in F} \gamma_{i, F}^{2}, \quad i \in[m],
$$

are sums of squares, which implies that $\phi_{\Delta}$ is a proper map, that is, compact sets have compact preimages under $\phi_{\Delta}$.

We will be interested in the situation when $\Delta$ is a simplicial complex on $[\mathrm{m}]$. In this case, the map $\phi_{\Delta}$ is never injective. It has fibers (preimages) of positive dimension unless the underlying graph is the empty graph.

Lemma 1.1. For any simplicial complex $\Delta$ on $[m]$ with underlying graph $G=$ $G(\Delta)$, the image of $\phi_{\Delta}$ is a closed full-dimensional semi-algebraic subset of $\mathbb{S}_{\succeq 0}^{m}(G)$.

Proof. If $i \neq j$ and $\{i, j\}$ is not an edge of $G$, then no face of $\Delta$ contains both $i$ and $j$. Hence, by (1.2), the image is a subset of $\mathbb{S}_{\succeq 0}^{m}(G)$. The image is semi-algebraic because $\phi_{\Delta}$ is a polynomial map, and it is closed because $\phi_{\Delta}$ is proper.

If $\Delta^{\prime} \subset \Delta$ is another simplicial complex with the same underlying graph then the image of $\phi_{\Delta^{\prime}}$ is contained in the image of $\phi_{\Delta}$. To show full dimension, we may thus assume that $\Delta$ is the complex whose facets are the edges of $G$. Using the shorthand $\gamma_{i}=\gamma_{i,\{i\}}$ and $\gamma_{i j}=\gamma_{i,\{i, j\}}$ in this special case, the non-zero coordinates of $\phi_{\Delta}$ are

$$
\phi_{\Delta}(\gamma)_{i j}= \begin{cases}\gamma_{i}^{2}+\sum_{k \in[m]:\{i, k\} \in \Delta} \gamma_{i k}^{2} & \text { if } i=j, \\ \gamma_{i j} \gamma_{j i} & \text { if } i \neq j .\end{cases}
$$

It is evident that there are no algebraic relations among these coordinates and, thus, the image is full-dimensional.

Example 1.2. Let $\Delta$ be the simplicial complex whose facets are the edges $\{1,2\}$ and $\{2,3\}$ of a three-chain. We have that

$$
\Gamma(\gamma)=\left(\begin{array}{ccccc}
\gamma_{1} & 0 & 0 & \gamma_{12} & 0 \\
0 & \gamma_{2} & 0 & \gamma_{21} & \gamma_{23} \\
0 & 0 & \gamma_{3} & 0 & \gamma_{32}
\end{array}\right)
$$

and

$$
\phi_{\Delta}(\gamma)=\left(\begin{array}{ccc}
\gamma_{1}^{2}+\gamma_{12}^{2} & \gamma_{12} \gamma_{21} & 0 \\
\gamma_{12} \gamma_{21} & \gamma_{2}^{2}+\gamma_{21}^{2}+\gamma_{23}^{2} & \gamma_{23} \gamma_{32} \\
0 & \gamma_{23} \gamma_{32} & \gamma_{3}^{2}+\gamma_{32}^{2}
\end{array}\right) .
$$

It can be shown that $\phi_{\Delta}$ is a surjective map onto the entire cone $\mathbb{S}_{\succeq 0}^{3}(G)$, which here comprises the tridiagonal positive semidefinite matrices. The surjectivity claim holds as a special case of Corollary 3.2 . 
As we describe in more detail in Section [6 the motivation for considering the parametrization $\phi_{\Delta}$ comes from statistics. The graphical cones $\mathbb{S}_{\succeq 0}^{m}(G)$ correspond to statistical models for the multivariate normal distribution; see [DP07, §2] and references therein. The parametrization $\phi_{\Delta}$ is particularly useful for tackling statistical problems in covariance graph models, which treat the cone $\mathbb{S}_{\succeq 0}(G)$ as a set of covariance matrices. The parametrization can be regarded as arising from constructions involving hidden or latent variables CW96, RS02. This connection can be exploited in particular for computation of maximum likelihood estimates and construction of prior distributions for Bayesian inference Bar08, PDB07. It also allows one to simplify the study of algebraic properties of graphical models based on mixed graphs; see [STD10.

In Example 1.2, the map $\phi_{\Delta}$ is surjective. However, it is known that surjectivity need not always hold. The following example has been given in the literature.

Example 1.3. Let $\Delta$ be the simplicial complex with facets $\{1,2\},\{1,3\}$ and $\{2,3\}$, and the complete graph $K_{3}$ as underlying graph. Now,

$$
\phi_{\Delta}(\gamma)=\left(\begin{array}{ccc}
\gamma_{1}^{2}+\gamma_{12}^{2}+\gamma_{13}^{2} & \gamma_{12} \gamma_{21} & \gamma_{13} \gamma_{31} \\
\gamma_{12} \gamma_{21} & \gamma_{2}^{2}+\gamma_{21}^{2}+\gamma_{23}^{2} & \gamma_{23} \gamma_{32} \\
\gamma_{13} \gamma_{31} & \gamma_{23} \gamma_{32} & \gamma_{3}^{2}+\gamma_{31}^{2}+\gamma_{32}^{2}
\end{array}\right)
$$

Suppose we are given a positive definite matrix $\Sigma=\left(\sigma_{i j}\right)$ in $\mathbb{S}_{\succ_{0}}^{3}\left(K_{3}\right)=\mathbb{S}_{\succ 0}^{3}$. Define the correlation matrix $R=\left(\rho_{i j}\right)$ with entries $\rho_{i j}=\sigma_{i j} / \sqrt{\sigma_{i i} \sigma_{j j}}$. The matrix $R$ is obtained by multiplying $\Sigma$ from the left and right with the diagonal matrix that has the entries $1 / \sqrt{\sigma_{i i}}$ on the diagonal. It follows that $\Sigma$ is in the image of $\phi_{\Delta}$ if and only if $R$ is in the image. For $R$ to be in the image, however, it needs to hold that

$$
\min \left\{\rho_{12}, \rho_{13}, \rho_{23}\right\} \leq \frac{1}{\sqrt{2}}
$$

see $\left[\mathrm{SRM}^{+} 98\right]$. Clearly, there are positive definite matrices in $\mathbb{S}_{\succeq 0}^{3}$ whose correlation matrices do not obey this condition.

Our Theorem 5.3 applies to this example and gives a semi-algebraic description of the image of $\phi_{\Delta}$. This description reveals that a positive definite matrix is in the image if and only if its correlation matrix $R$ satisfies

$$
1-\rho_{12}^{2}-\rho_{13}^{2}-\rho_{23}^{2}-2 \rho_{12} \rho_{13} \rho_{23} \geq 0 .
$$

If $\rho_{12}, \rho_{13}, \rho_{23}>1 / 2$, then the left hand side in (1.5) is smaller than $1-3 / 4-2 / 8=0$. Hence, one may replace $1 / \sqrt{2}$ by $1 / 2$ in the necessary condition in (1.4), which can also be seen directly. If $R=\phi_{\Delta}(\gamma)$ has diagonal entries 1 , then summing the diagonal entries gives

$$
3=\sum_{i=1}^{3} \gamma_{i}^{2}+\sum_{1 \leq i<j \leq 3}\left(\gamma_{i j}^{2}+\gamma_{j i}^{2}\right)=\sum_{i=1}^{3} \gamma_{i}^{2}+\sum_{1 \leq i<j \leq 3}\left(\gamma_{i j}-\gamma_{j i}\right)^{2}+\sum_{1 \leq i<j \leq 3} 2 \gamma_{i j} \gamma_{j i},
$$

so we must have $\rho_{i j}=\gamma_{i j} \gamma_{j i} \leq 1 / 2$ for some $i, j$.

This paper explores in detail the images of the maps $\phi_{\Delta}$, which we denote by $\operatorname{im}\left(\phi_{\Delta}\right)$. In Section 2, we show that the image is always a convex cone and we describe its extreme rays. In Section 3, we prove that surjectivity of the map can only be achieved if $\Delta$ is the clique complex of a chordal (or decomposable) graph. Section 4 collects results relevant for passing to submatrices and Schur 
complements. In Section 5, we derive the semi-algebraic description of the image when the underlying graph is a chordless cycle. The connection to statistical models is reviewed in Section 6 .

\section{Convexity}

The set $\mathbb{S}_{\succeq 0}^{m}$ of positive semidefinite $m \times m$ matrices forms a full-dimensional convex cone in the $m(m+1) / 2$ dimensional vector space of $m \times m$ symmetric matrices. A ray of $\mathbb{S}_{\succ 0}^{m}$ is the set of non-negative scalar multiples of some non-zero matrix in $\mathbb{S}_{\succeq 0}^{m}$. An extreme ray is a ray that cannot be written as a positive linear combination of two distinct rays. The extreme rays of $\mathbb{S}_{\succeq 0}^{m}$ are given by the positive semidefinite matrices of rank 1 . Hence, $\mathbb{S}_{\succ 0}^{m}$ is the convex hull of its rank 1 elements.

For $F \subseteq[m]$, let $\mathbb{S}_{\succ 0}^{m}(F)$ be the convex cone of positive semidefinite matrices that have zeros outside the $F \times F$ submatrix.

Theorem 2.1. For any simplicial complex $\Delta$ on $[m]$, the image of $\phi_{\Delta}$ is a convex cone. The matrices on the extreme rays of the image are the rank one matrices that are in $\mathbb{S}_{\succeq 0}^{m}(F)$ for some face $F \in \Delta$. In other words, $\operatorname{im}\left(\phi_{\Delta}\right)=\sum_{F \in \Delta} \mathbb{S}_{\succeq 0}^{m}(F)$.

Proof. Elements of the image of the map $\phi_{\Delta}$ are of the form

$$
\sum_{F \in \Delta} \Gamma(\gamma)_{F} \Gamma(\gamma)_{F}^{T}
$$

where $\Gamma(\gamma)_{F}$ is the column of $\Gamma(\gamma)$ corresponding to face $F$. This column can be any vector in $\mathbb{R}^{m}$ that has $i$-th entry zero for each $i \notin F$. It is clear that the image of $\phi_{\Delta}$ is closed under positive scaling. We will show that it is closed under addition, by induction on the maximal cardinality of a face in $\Delta$.

If all faces have size 1 then the image of $\phi_{\Delta}$ consists of all positive semidefinite diagonal matrices and is convex. Let $F$ be a facet of $\Delta$ and suppose it has cardinality at least 2. Consider the matrix

$$
\Sigma=\Gamma(\gamma)_{F} \Gamma(\gamma)_{F}^{T}+\Gamma\left(\gamma^{\prime}\right)_{F} \Gamma\left(\gamma^{\prime}\right)_{F}^{T}
$$

and its Cholesky decomposition $\Sigma=L L^{T}$, where $L$ is a lower triangular matrix. Since $\Sigma \in \mathbb{S}_{\succeq 0}^{m}(F)$, each column of the Cholesky factor $L$ has support in $F$. In fact only the first column of $L$ may have support equal to $F$; denote this column by $L_{1}$. All other columns of $L$ have support strictly smaller than $F$. These smaller supports correspond to subfaces of $F$, so they are in $\Delta$. Hence, $\Sigma$ is the sum of $L_{1} L_{1}^{T}$ and an element in the image of $\phi_{\Delta \backslash\{F\}}$. (Removing a facet leaves us with another simplicial complex.) Repeating this process for all other faces of maximal cardinality in $\Delta$ and using the inductive hypothesis, we see that the image of $\phi_{\Delta}$ is closed under addition.

Suppose a non-zero matrix $\Sigma$ is on an extreme ray of the convex cone $\operatorname{im}\left(\phi_{\Delta}\right)$. Then $\Sigma=\Sigma_{1}+\Sigma_{2}$ for some non-zero and distinct matrices $\Sigma_{1}, \Sigma_{2}$ in the same cone implies that both $\Sigma_{1}$ and $\Sigma_{2}$ are scalar multiples of $\Sigma$. From the definition, any element in the image of $\phi_{\Delta}$ is a sum of rank one matrices in it, so only rank one matrices can be on the extreme rays. Moreover, any rank one positive semidefinite matrix is on an extreme ray of $\mathbb{S}_{\succeq 0}^{m}$, so it is also on an extreme ray of the convex subcone $\operatorname{im}\left(\phi_{\Delta}\right)$ that contains it. A rank one matrix in $\operatorname{im}\left(\phi_{\Delta}\right)$ is of the form $v v^{T}$ for some vector $v \in \mathbb{R}^{m}$ whose support $F$ is a face of $\Delta$. Hence, $v v^{T} \in \mathbb{S}_{\succeq 0}^{m}(F)$. 
A clique in an undirected graph $G$ with vertex set $[m]$ is a subset $F \subseteq[m]$ such that for any pair of distinct vertices $i, j \in F,\{i, j\}$ is in $E(G)$. The set of all cliques in $G$ forms a simplicial complex on $[m]$ and is called the clique complex of $G$.

Corollary 2.2. Let $\Delta$ be a simplicial complex on $[m]$ with underlying graph $G$. Then the extreme rays of the image of $\phi_{\Delta}$ consist of all rank one matrices in $\mathbb{S}_{\succeq 0}^{m}(G)$ if and only if $\Delta$ consists of all the cliques in $G$.

\section{SurJectivity}

The maximal rank of a matrix lying on an extreme ray of $\mathbb{S}_{\succeq 0}^{m}(G)$ is called the sparsity order of the graph $G$ and denoted $\operatorname{ord}(G)$. A subgraph $\bar{H}$ of $G$ is called an induced subgraph if for all pairs of vertices $i, j$ in $H,\{i, j\} \in E(H) \Longleftrightarrow\{i, j\} \in$ $E(G)$. A graph is called chordal if it does not contain any chordless cycle of size more than three as an induced subgraph. The following results are known in the literature AHMR88, HPR89, Lau01.

Theorem 3.1. For a graph $G$ with $m$ vertices,

(i) $1 \leq \operatorname{ord}(G) \leq m-2$,

(ii) $\operatorname{ord}(G)=1$ if and only if $G$ is chordal,

(iii) $\operatorname{ord}(G)=m-2$ if and only if $m \leq 3$ or $G$ is a chordless cycle, and

(iv) if $H$ is an induced subgraph of $G$, then $\operatorname{ord}(H) \leq \operatorname{ord}(G)$.

These results readily allow one to characterize when the parametrization $\phi_{\Delta}$ fills all of the graphical cone $\mathbb{S}_{\succeq 0}^{m}(G)$.

Corollary 3.2. Let $\Delta$ be a simplicial complex and $G$ a graph on $[m]$. The map $\phi_{\Delta}$ is surjective onto $\mathbb{S}_{\succeq 0}^{m}(G)$ if and only if $G$ is chordal and $\Delta$ is its clique complex.

Proof. (Sufficiency) If $\Delta$ contains all cliques in $G$, then $\operatorname{im}\left(\phi_{\Delta}\right)$ contains all rank one matrices in $\mathbb{S}_{\succeq 0}^{m}(G)$. If $G$ is chordal, then its sparsity order is one, so $\mathbb{S}_{\succeq 0}^{m}(G)$ is generated by rank one matrices in it. Hence, $\operatorname{im}\left(\phi_{\Delta}\right)=\mathbb{S}_{\succ 0}^{m}(G)$ and $\phi_{\Delta}$ is surjective.

(Necessity) First note that the image of $\phi_{\Delta}$ is a subset of $\mathbb{S}_{\succeq 0}^{m}(G)$ only if all sets in $\Delta$ are cliques of $G$.

Let $\Delta$ be the clique complex of $G$. If $G$ is not chordal, then there is an induced subgraph that is a chordless cycle of size at least 4 . So $\operatorname{ord}(G) \geq 2$, and there is an extreme ray of $\mathbb{S}_{\succeq 0}^{m}(G)$ containing matrices of rank at least two. This ray is not in the convex cone $\operatorname{im}\left(\phi_{\Delta}\right)$, so $\phi_{\Delta}$ is not surjective. It follows that $\phi_{\Delta^{\prime}}$ is not surjective for any (arbitrary) subset $\Delta^{\prime}$ of $\Delta$.

Suppose $\Delta$ does not contain a clique $F$ in $G$. Let $v \in \mathbb{R}^{m}$ be a vector with support $F$. Then $v v^{T}$ is a rank one element of $\mathbb{S}_{\succeq 0}^{m}(G)$. It lies in an extreme ray of $\mathbb{S}_{\succeq 0}^{m}(G)$ because it lies in an extreme ray of the larger cone $\mathbb{S}_{\succeq 0}^{m}$. Hence, it cannot be written as a sum of other elements in $\mathbb{S}_{\succeq 0}^{m}(G)$, so it is not in $\operatorname{im}\left(\phi_{\Delta}\right)$, and $\phi_{\Delta}$ is not surjective.

Remark 3.3. The sufficiency of the condition in Corollary 3.2 can also be proved by using the Cholesky decomposition to compute a point in the fiber $\phi_{\Delta}^{-1}(\Sigma)$ of a matrix $\Sigma \in \mathbb{S}_{\succ 0}^{m}(G)$. The vertices of a chordal graph $G$ can be brought into a perfect elimination ordering, which ensures sparsity of the lower-triangular Cholesky factor; see for example [PPS89, Thm. 2.4]. Suppose the original vertices $1, \ldots, m$ are already in such an order. Then $\Sigma=L L^{T}$ for a lower-triangular matrix $L=\left(l_{i j}\right)$ 
with $l_{i j}=0$ when $i \neq j$ and $\{i, j\}$ is not an edge of $G$. The support of each column of $L$ is thus a clique in $G$. It follows that $\Sigma \in \operatorname{im}\left(\phi_{\Delta}\right)$.

The necessity of the chordality condition in Corollary 3.2 also follows from our semi-algebraic characterization of $\operatorname{im}\left(\phi_{\Delta}\right)$ when $\Delta$ is the clique complex of a chordless cycle; see Section 5 that also gives an example of a matrix not in the image.

In the statistical literature, the parametrization $\phi_{\Delta}$ is most commonly considered for a simplicial complex $\Delta$ given by the edges of a graph. The parametrization for such an edge complex is surjective only for chordal graphs whose cliques are of cardinality at most two. This means that there may not be any cycles.

Corollary 3.4. The edge complex $\Delta$ of a graph $G$ yields a surjective parametrization $\phi_{\Delta}$ of $\mathbb{S}_{\succeq 0}^{m}(G)$ if and only if $G$ is a forest (has no cycles).

\section{Submatrices and Schur Complements}

For a simplicial complex $\Delta$ on $[m]$ and a subset $A \subseteq[m]$, define the induced subcomplex $\Delta_{A}=\{F \in \Delta: F \subseteq A\}$.

Lemma 4.1. Let $\Delta$ be a simplicial complex on $[\mathrm{m}]$. If $\Sigma$ is a matrix in the image of $\phi_{\Delta}$, then all proper principal submatrices $\Sigma_{A, A}, A \subset[m]$, are in the image of the respective induced subcomplex $\phi_{\Delta_{A}}$.

Proof. Write $\Sigma=\Gamma(\gamma) \Gamma(\gamma)^{T}$. Let $\Gamma_{A}(\gamma)$ be the submatrix of $\Gamma(\gamma)$ obtained by removing all rows with index not in $A$. Then $\Sigma_{A, A}=\Gamma_{A}(\gamma) \Gamma_{A}(\gamma)^{T}+\operatorname{diag}\left(\gamma^{\prime}\right)$ where $\gamma_{i}^{\prime}=\sum_{F \in \Delta \backslash \Delta_{A}} \gamma_{i, F}^{2}$. The matrix $\Gamma_{A}(\gamma) \Gamma_{A}(\gamma)^{T}$ is in the image of $\phi_{\Delta_{A}}$, and so is the diagonal matrix $\operatorname{diag}\left(\gamma^{\prime}\right)$. By convexity (Theorem 2.1), $\Sigma_{A, A} \in \operatorname{im}\left(\phi_{\Delta_{A}}\right)$.

The converse of the lemma does not hold. If $\Delta$ is the edge complex of a chordless cycle, then any matrix in $\mathbb{S}_{\succ 0}^{m}(G)$ has all of its proper principal submatrices in the image of the corresponding map $\phi_{\Delta_{A}}$, but $\operatorname{im}\left(\phi_{G}\right) \subsetneq \mathbb{S}_{\succ 0}^{m}(G)$ by Corollary 3.2.

For a square matrix $M$ partitioned as

$$
M=\left(\begin{array}{cc}
A & B \\
C & D
\end{array}\right)
$$

the Schur complement of a non-singular submatrix $D$ in $M$ is defined as $M / D:=$ $A-B D^{-1} C$. If $M$ is symmetric positive semidefinite, then so is $M / D$. If $D$ is further partitioned as

$$
D=\left(\begin{array}{cc}
E & F \\
G & H
\end{array}\right),
$$

and $H$ and $D / H$ are non-singular, then the following quotient formula holds: $M / D=(M / H) /(D / H)$. Proofs can be found in textbooks on matrix theory.

For a graph $G=(V, E)$ and a proper subset of vertices $U \subset V$, define a new graph $G / U$ on vertex set $V \backslash U$ as follows. A pair $\{i, j\} \subseteq V \backslash U$ is an edge in $G / U$ if $\{i, j\}$ is an edge in $G$ or there is a path between $i$ and $j$ in $G$ through vertices in $U$. For a simplicial complex $\Delta$ on ground set $V$, define a new simplicial complex $\Delta / U$ on $V \backslash U$ where a set $A \subseteq V \backslash U$ forms a face if it is a face in $\Delta$ or there exists a sequence of distinct elements $u_{1}, u_{2}, \ldots, u_{k} \in U$ and distinct faces $F_{1}, \ldots, F_{k+1} \in \Delta$ such that $u_{i} \in F_{i} \cap F_{i+1}$ and $A=\bigcup_{i=1}^{k+1} F_{i} \backslash U$. If the faces of $\Delta$ form cliques in $G$, then the faces of $\Delta / U$ form cliques in $G / U$. 
Proposition 4.2. Let $\Delta$ be a simplicial complex on $[m]$ and $U \subsetneq[m]$ a proper subset of nodes. If $\Sigma$ is in the image of $\phi_{\Delta}$ and $\Sigma_{U, U}$ is non-singular, then the Schur complement $\Sigma / \Sigma_{U, U}$ is in the image of $\phi_{\Delta / U}$.

Proof. If $U^{\prime}$ is a non-empty proper subset of $U$, then by the quotient formula we have $\Sigma / \Sigma_{U, U}=\left(\Sigma / \Sigma_{U^{\prime}, U^{\prime}}\right) /\left(\Sigma_{U, U} / \Sigma_{U^{\prime}, U^{\prime}}\right)$. Moreover, $\Delta / U=\left(\Delta / U^{\prime}\right) /\left(U \backslash U^{\prime}\right)$ by construction. Therefore, it suffices to prove the assertion when $U$ consists of only one vertex $u$. We call a face $F$ of the complex $\Delta / u:=\Delta /\{u\}$ original if $F$ is also a face of $\Delta$ and induced if $F=\left(F_{1} \cup F_{2}\right) \backslash\{u\}$ for a pair of distinct faces $F_{1}, F_{2}$ of $\Delta$ that both contain $u$. Note that a face can be both original and induced.

Let $\Sigma=\Gamma(\gamma) \Gamma(\gamma)^{T}$ be in the image of $\phi_{\Delta}$. Define as follows a new matrix $\Gamma_{\Delta / u}\left(\gamma^{\prime}\right)=\left[\gamma_{v, F}^{\prime}\right]$ whose rows and columns are indexed by the vertices and the induced faces of $\Delta / u$, respectively. Fix an arbitrary total order " $\leq$ " on the faces of $\Delta$. For the induced face $F=\left(F_{1} \cup F_{2}\right) \backslash\{u\}$ given by a pair of faces $F_{1}<F_{2}$ of $\Delta$ with $u \in F_{1} \cap F_{2}$, let

$$
\gamma_{i F}^{\prime}=\left(\gamma_{i F_{1}} \gamma_{u F_{2}}-\gamma_{i F_{2}} \gamma_{u F_{1}}\right) / \sqrt{\sigma_{u u}} .
$$

Here, $\gamma_{i F_{j}}$ is shorthand for $\gamma_{i, F_{j}}$ and $\gamma_{i F_{j}}=0$ if $i \notin F_{j}$.

Let $A=V \backslash\{u\}$. We now show the following:

$$
\Sigma / \Sigma_{u, u}:=\Sigma_{A, A}-\frac{\Sigma_{A, u} \Sigma_{u, A}}{\sigma_{u u}}=\Gamma_{\Delta_{A}}(\gamma) \Gamma_{\Delta_{A}}(\gamma)^{T}+\Gamma_{\Delta / u}\left(\gamma^{\prime}\right) \Gamma_{\Delta / u}\left(\gamma^{\prime}\right)^{T}
$$

where $\Gamma_{\Delta_{A}}(\gamma)$ is the submatrix of $\Gamma(\gamma)$ with rows and columns indexed respectively by $A$ and faces $F \in \Delta$ contained in $A$. The $i j$-entry on the right hand side is

$$
\begin{aligned}
& \sum_{\substack{F \in \Delta / u \\
F \text { original }}} \gamma_{i F} \gamma_{j F}+\sum_{\substack{F \in \Delta / u \\
F \text { induced }}} \gamma_{i F}^{\prime} \gamma_{j, F}^{\prime} \\
= & \sum_{\substack{F \in \Delta \\
u \notin F}} \gamma_{i F} \gamma_{j F}+\frac{1}{\sigma_{u u}} \sum_{\substack{F_{1}<F_{2} \in \Delta \\
u \in F_{1} \cap F_{2}}}\left(\gamma_{i F_{1}} \gamma_{u F_{2}}-\gamma_{i F_{2}} \gamma_{u F_{1}}\right)\left(\gamma_{j F_{1}} \gamma_{u F_{2}}-\gamma_{j F_{2}} \gamma_{u F_{1}}\right) \\
= & \sum_{\substack{F \in \Delta \\
u \notin F}} \gamma_{i F} \gamma_{j F}+\frac{1}{\sigma_{u u}} \sum_{\substack{F_{1} \neq F_{2} \in \Delta \\
u \in F_{1} \cap F_{2}}}\left(-\gamma_{i F_{1}} \gamma_{j F_{2}} \gamma_{u F_{1}} \gamma_{u F_{2}}+\gamma_{i F_{1}} \gamma_{j F_{1}} \gamma_{u F_{2}}^{2}\right) \\
= & \sum_{\substack{F \in \Delta \\
u \notin F}} \gamma_{i F} \gamma_{j F}+\frac{1}{\sigma_{u u}} \sum_{\substack{F_{1}, F_{2} \in \Delta \\
u \in F_{1} \cap F_{2}}}\left(-\gamma_{i F_{1}} \gamma_{j F_{2}} \gamma_{u F_{1}} \gamma_{u F_{2}}+\gamma_{i F_{1}} \gamma_{j F_{1}} \gamma_{u F_{2}}^{2}\right) \\
= & \sum_{\substack{F \in \Delta \\
u \notin F}} \gamma_{i F} \gamma_{j F}+\frac{1}{\sigma_{u u}} \sum_{\substack{F_{1} \in \Delta \\
u \in F_{1}}} \gamma_{i F_{1}} \gamma_{j F_{1}} \sum_{\substack{F_{2} \in \Delta \\
u \in F_{2}}} \gamma_{u F_{2}}^{2}-\frac{1}{\sigma_{u u}} \sum_{\substack{F_{1}, F_{2} \in \Delta \\
u \in F_{1} \cap F_{2}}} \gamma_{i F_{1}} \gamma_{j F_{2}} \gamma_{u F_{1}} \gamma_{u F_{2}} \\
= & \sum_{F \in \Delta} \gamma_{i F} \gamma_{j F}-\frac{1}{\sigma_{u u}} \sum_{\substack{F_{1}, F_{2} \in \Delta \\
u \in F_{1} \cap F_{2}}} \gamma_{i F_{1}} \gamma_{j F_{2}} \gamma_{u F_{1}} \gamma_{u F_{2}},
\end{aligned}
$$

which is equal to the $i j$-entry on the left hand side because $\sigma_{i j}=\sum_{F \in \Delta} \gamma_{i F} \gamma_{j F}$. From (4.1) and the convexity of $\operatorname{im}\left(\phi_{\Delta / u}\right)$ shown in Theorem [2.1, it follows that Schur complement $\Sigma / \Sigma_{u, u}$ is in the image of $\phi_{\Delta / u}$.

The converse of Proposition 4.2 cannot hold in general. Let $G$ be a 3 -cycle and consider $\phi_{G}$ given by the edge complex of $G$. Then any Schur complement $\Sigma / \Sigma_{u, u}$ of $\Sigma \in \mathbb{S}_{\succ 0}^{m}(G)$ is in $\operatorname{im}\left(\phi_{G /\{u\}}\right)$, but not every such matrix $\Sigma$ is in $\operatorname{im}\left(\phi_{G}\right)$. 


\section{ChORdLess CYCLES}

Let $C_{m}$ be the chordless $m$-cycle with edges $\{1,2\},\{2,3\}, \ldots,\{1, m\}$. Excluding trivial cases, assume that $m \geq 3$. Let $\Delta$ be the simplicial complex whose facets are the edges of $C_{m}$, and define $\phi_{C_{m}}=\phi_{\Delta}$. For $m \geq 4$, there is no other simplicial complex $\Delta^{\prime}$ that gives a parametrization $\phi_{\Delta^{\prime}}$ whose image is a full-dimensional subset of $\mathbb{S}_{\succeq 0}^{m}\left(C_{m}\right)$. In this section we give a semi-algebraic description of $\operatorname{im}\left(\phi_{C_{m}}\right)$.

We begin with a simple yet important observation. For a symmetric matrix $\Sigma \in \mathbb{S}^{m}$ and two distinct indices $i, j \in[m]$, define $\Sigma^{(i j)}$ to be the symmetric matrix obtained by negating the $(i, j)$ and $(j, i)$ entries of $\Sigma$.

Lemma 5.1. Suppose $\Sigma \in \mathbb{S}_{\succeq 0}^{m}$ is a positive semidefinite matrix, and $\Delta$ is the edge complex of a graph. If $i \neq j$ and $\{i, j\} \in \Delta$, then $\Sigma$ is in the image of $\phi_{\Delta}$ if and only if $\Sigma^{(i j)}$ is in $\operatorname{im}\left(\phi_{\Delta}\right)$.

Proof. If $\Sigma=\phi_{\Delta}(\gamma)$, then $\Sigma^{(i j)}=\phi_{\Delta}(\bar{\gamma})$, where $\bar{\gamma}$ is identical to $\gamma$ except for the single entry $\gamma_{i j}=\gamma_{i,\{i, j\}}$ that is replaced by its negative $-\gamma_{i j}$. The entry $\gamma_{j i}$ remains unchanged.

Note that Lemma 5.1 immediately yields the necessary condition stated in (1.5) in Example 1.3 about $C_{3}=K_{3}$, the complete graph on 3 nodes. The lemma can also be used to give an explicit example of a matrix not in the image of the parametrization for $C_{m}, m \geq 3$.

Example 5.2. For $m \geq 3$, define the symmetric $m \times m$ matrix

$$
\Sigma(\rho)=\left(\begin{array}{cccccc}
1 & \frac{1}{2} & & & & \frac{1}{2} \rho \\
\frac{1}{2} & 1 & \frac{1}{2} & & & \\
& \ddots & \ddots & \ddots & \ddots & \\
& & & \frac{1}{2} & 1 & \frac{1}{2} \\
\frac{1}{2} \rho & & & & \frac{1}{2} & 1
\end{array}\right),
$$

where all omitted entries are zero such that $\Sigma(\rho) \in \mathbb{S}^{m}\left(C_{m}\right)$. Omitting the $m$-th row and column of $\Sigma(\rho)$ yields a positive definite tridiagonal matrix. Hence, $\Sigma(\rho)$ is in the graphical cone $\mathbb{S}_{\succeq 0}^{m}\left(C_{m}\right)$ if and only if $\operatorname{det}(\Sigma(\rho)) \geq 0$. Using Laplace expansions and the recursive formula for the determinant of a tridiagonal matrix, one can show that

$$
\operatorname{det} \Sigma(\rho)= \begin{cases}\frac{1}{2^{m}}[m+1-(m-1) \rho](1+\rho) & \text { if } m \text { is odd } \\ \frac{1}{2^{m}}[m+1+(m-1) \rho](1-\rho) & \text { if } m \text { is even }\end{cases}
$$

If $m$ is odd, choose $\rho \in(1,1+2 /(m-1))$. If $m$ is even, choose $\rho \in(-1-2 /(m-$ $1),-1)$. Then the determinant of $\Sigma(\rho)$ is positive but the determinant of $\Sigma(-\rho)$ is negative. Since $\Sigma(\rho)^{(1 m)}=\Sigma(-\rho)$, it follows from Lemma 5.1] that $\Sigma(\rho)$ is in $\mathbb{S}_{\succeq 0}^{m}\left(C_{m}\right)$ but not in the image of $\phi_{C_{m}}$.

We now state the main result of this section, a semi-algebraic description of the image of $\phi_{C_{m}}$. Let $\mathcal{M}\left(C_{m}\right)$ be the collection of all (not necessarily maximal) matchings of $C_{m}$. A matching is any set $M$ of pairwise disjoint edges, that is, $\{i, j\},\{k, l\} \in M$ implies that $\{i, j\} \cap\{k, l\}=\emptyset$. For $M \in \mathcal{M}\left(C_{m}\right)$, we write $[m] \backslash M$ to denote the set of nodes not incident to any edge in $M$. If $\Sigma=\left(\sigma_{i j}\right)$ is a 
matrix in $\mathbb{S}_{\succeq 0}^{m}\left(C_{m}\right)$ then its determinant can be expanded as

$$
\operatorname{det}(\Sigma)=(-1)^{m+1} \cdot 2 \prod_{i=1}^{m} \sigma_{i, i+1}+\sum_{M \in \mathcal{M}\left(C_{m}\right)}(-1)^{|M|} \prod_{\{i, j\} \in M} \sigma_{i j}^{2} \prod_{i \in[m] \backslash M} \sigma_{i i} ;
$$

compare CDS95, $\$ 1.4$, eqn. (1.42)]. In the first term, which corresponds to the entire cycle $C_{m}$, the indices are read modulo $m$ such that $\sigma_{m, m+1} \equiv \sigma_{1 m}$. The following theorem is the main result of this section.

Theorem 5.3. A matrix $\Sigma=\left(\sigma_{i j}\right) \in \mathbb{S}_{\succeq 0}^{m}\left(C_{m}\right)$ is in the image of $\phi_{C_{m}}$ if and only if

$$
\sum_{M \in \mathcal{M}\left(C_{m}\right)}(-1)^{|M|} \prod_{\{i, j\} \in M} \sigma_{i j}^{2} \prod_{i \in[m] \backslash M} \sigma_{i i} \geq 2 \prod_{i=1}^{m}\left|\sigma_{i, i+1}\right| .
$$

Proof. (Necessity) A matrix $\Sigma \in \operatorname{im}\left(\phi_{C_{m}}\right)$ is positive semidefinite and thus has a non-negative determinant. Pick any edge in $C_{m}$, say $\{1,2\}$. Then, by Lemma 5.1 $\Sigma^{(12)}$ is positive semidefinite. Hence,

$$
\begin{aligned}
& \min \left\{\operatorname{det}(\Sigma), \operatorname{det}\left(\Sigma^{(12)}\right)\right\}= \\
& \quad-2 \prod_{i=1}^{m}\left|\sigma_{i, i+1}\right|+\sum_{M \in \mathcal{M}\left(C_{m}\right)}(-1)^{|M|} \prod_{\{i, j\} \in M} \sigma_{i j}^{2} \prod_{i \in[m] \backslash M} \sigma_{i i} \geq 0 .
\end{aligned}
$$

(Sufficiency) We need to show that under the assumed condition on $\Sigma \in \mathbb{S}_{\succ 0}\left(C_{m}\right)$, the equation system $\phi_{C_{m}}(\gamma)=\Sigma$ has a feasible solution $(\gamma)$ in $(\mathbb{R})^{V} \times\left(\mathbb{R}^{2}\right)^{E}$. Our proof will show that $\gamma_{i,\{i\}}$ does not play an important role and can simply be set to zero. Since $\operatorname{im}\left(\phi_{C_{m}}\right)$ is closed and full-dimensional in $\mathbb{S}_{\succeq 0}(G)$, it suffices to show that a dense (Zariski open) subset of points $\Sigma$ satisfying the necessity condition is contained in $\operatorname{im}\left(\phi_{C_{m}}\right)$. We show that for positive definite $\Sigma$, any complex solution $\gamma\left(\right.$ with $\gamma_{i,\{i\}}=0$ for all $\left.i=1, \ldots, m\right)$ is in fact real and thus feasible (Lemma 5.5). The proof is completed by demonstrating the existence of complex solutions for generic $\Sigma$ (Lemma [5.6).

The following is an immediate consequence of Theorem 5.3

Corollary 5.4. For a positive semidefinite matrix $\Sigma=\left(\sigma_{i j}\right)$ in $\mathbb{S}_{\succeq 0}\left(C_{m}\right)$ the following are equivalent:

(1) $\Sigma$ is in the image of $\phi_{C_{m}}$.

(2) $\Sigma^{(i j)}$ is positive semidefinite for all edges $i j \in C_{m}$.

(3) $\Sigma^{(i j)}$ is positive semidefinite for some edge ij $\in C_{m}$.

Proof. By Lemma 5.1, we have (1) implies (2). It is obvious that (2) implies (3). If both $\Sigma$ and $\Sigma^{(i j)}$ have non-negative determinants for some edge $i j \in C_{m}$, then the inequality in Theorem 5.3 is satisfied, so we have (3) implies (1).

The semi-algebraic condition from Theorem 5.3 is easily verified, and we can use it to compute the spherical volume of the cone $\operatorname{im}\left(\phi_{C_{m}}\right)$ by Monte Carlo integration. Table 1 shows which fraction of the cone $\mathbb{S}_{\succeq 0}\left(C_{m}\right)$ is covered by the image of $\phi_{C_{m}}$, when quantifying this by the ratio of the spherical volumes of the two cones. The rounded ratios were computed by simulating 100,000 matrices in $\mathbb{S}_{\succeq 0}\left(C_{m}\right)$. In terms of the ratio of spherical volumes, the difference between $\operatorname{im}\left(\phi_{C_{m}}\right)$ and $\mathbb{S}_{\succeq 0}\left(C_{m}\right)$ is largest for $m=3$ and becomes rather minor for $m=6,7$. 
TABLE 1. Spherical volume of the image of $\phi_{C_{m}}$ as a fraction of the spherical volume of the cone $\mathbb{S}_{\succeq 0}\left(C_{m}\right)$.

\begin{tabular}{cccccc}
\hline \hline$m$ & 3 & 4 & 5 & 6 & 7 \\
Vol & 0.78 & 0.90 & 0.95 & 0.98 & 0.99 \\
\hline
\end{tabular}

The remainder of the section is devoted to the details of the proof of the sufficiency of the condition in Theorem 5.3 As mentioned above, our approach is to study the equation system $\phi_{\Delta}(\gamma)=\Sigma$ for a given matrix $\Sigma=\left(\sigma_{i j}\right) \in \mathbb{S}_{\succeq 0}(G)$, which is treated as a parameter to the system. For $\Delta=E\left(C_{m}\right)$, the equations take the form:

$$
\begin{aligned}
\gamma_{i,\{i\}}^{2}+\gamma_{i, i-1}^{2}+\gamma_{i, i+1}^{2} & =\sigma_{i i}, & & i=1, \ldots, m, \\
\gamma_{i-1, i} \gamma_{i, i-1} & =\sigma_{i-1, i}, & & i=1, \ldots, m .
\end{aligned}
$$

where $\gamma_{i, j}$ denotes $\gamma_{i,\{i, j\}}$ and indices are read modulo $m$ such that $0 \equiv m$ and $1 \equiv m+1$. The $2 m$ equations in (5.2a) and (5.2b) involve $3 m$ unknowns and have an $m$-dimensional solution set in $\mathbb{C}^{3 m}$. In the sequel we simply omit the unknowns $\gamma_{i,\{i\}}$ from the system. In other words, we study the $2 m$ equations in $2 m$ unknowns:

$$
\begin{aligned}
\gamma_{i, i-1}^{2}+\gamma_{i, i+1}^{2} & =\sigma_{i i}, & & i=1, \ldots, m, \\
\gamma_{i-1, i} \gamma_{i, i-1} & =\sigma_{i-1, i}, & i & =1, \ldots, m .
\end{aligned}
$$

We will show that system (5.3a)-(5.3b) has a real solution if $\Sigma$ satisfies the condition from Theorem 5.3, which will imply that the system (5.2a)-(5.2a) has a real solution, too. Somewhat surprisingly it suffices to argue that they have a complex solution, as is made precise in the next lemma.

Lemma 5.5. If a positive definite matrix $\Sigma \in \mathbb{S}_{\succeq 0}\left(C_{m}\right)$ satisfies the necessary condition from Theorem [5.3, then all complex solutions to the equations (5.3a)(5.36) are in fact real.

Proof. Suppose the vector $\gamma \in\left(\mathbb{C}^{2}\right)^{E}$ provides a solution to (5.3a)- $5.3 \mathrm{~b}$. Fill the $2 m$ unknowns in a matrix $\Gamma(\gamma) \in \mathbb{C}^{V \times E} \simeq \mathbb{C}^{m \times m}$ as in (1.1). Note that we are setting $\gamma_{i,\{i\}}=0$ for all $i=1, \ldots, m$, so the columns in $\Gamma(\gamma)$ corresponding to the singleton faces $\{1\}, \ldots,\{m\}$ are zero and can be omitted. Augment $\Gamma(\gamma)$ to an $(m+1) \times m$ matrix $\Gamma_{(12)}$ by adding the vector $\left(\gamma_{12}, 0, \ldots, 0\right)$ as a first row. Based on the equations (5.3a)- (5.3b),

$$
\Gamma_{(12)} \Gamma_{(12)}^{T}=\left(\begin{array}{cccccc}
\gamma_{12}^{2} & \gamma_{12}^{2} & \sigma_{12} & & & \\
\gamma_{12}^{2} & \sigma_{11} & \sigma_{12} & & \sigma_{1 m} & \\
\sigma_{12} & \sigma_{12} & \sigma_{22} & \sigma_{23} & & \\
& & \sigma_{23} & \sigma_{33} & \ddots & \\
& & & \ddots & \ddots & \sigma_{m-1, m} \\
& \sigma_{1 m} & & & \sigma_{m-1, m} & \sigma_{m m}
\end{array}\right)
$$

with blank entries being zero. The $(m+1) \times(m+1)$ matrix $\Gamma_{(12)} \Gamma_{(12)}^{T}$ has rank at most $m$, and thus its determinant vanishes. Therefore, $\gamma_{12}$ has to satisfy the quartic equation

$$
\operatorname{det}\left(\Gamma_{(12)} \Gamma_{(12)}^{T}\right)=a \gamma_{12}^{4}+b \gamma_{12}^{2}+c=0
$$


By expanding the determinant of (5.4) along the first row (or column), the coefficients in (5.5) are found to be:

$$
\begin{aligned}
& a=-\operatorname{det}\left(\Sigma_{[m] \backslash\{1\},[m] \backslash\{1\}}\right), \\
& b=\operatorname{det}(\Sigma)+2 \sigma_{12}^{2} \operatorname{det}\left(\Sigma_{[m] \backslash\{1,2\},[m] \backslash\{1,2\}}\right)+(-1)^{m} \cdot 2 \prod_{i=1}^{m} \sigma_{i-1, i}, \\
& c=-\sigma_{12}^{2} \operatorname{det}\left(\Sigma_{[m] \backslash\{2\},[m] \backslash\{2\}}\right) .
\end{aligned}
$$

Note that the third term in $b$ cancels out a term in $\operatorname{det}(\Sigma)$; recall (5.1). It remains to argue that under the assumption on $\Sigma$, the quartic in (5.5) has only real solutions. Define $\Sigma^{(12)}$ by negating $\sigma_{12}$ as in Lemma [5.1] Then the assumption on $\Sigma$ implies that both $\operatorname{det}(\Sigma)$ and $\operatorname{det}\left(\Sigma^{(12)}\right)$ are positive.

First, we claim that the discriminant $b^{2}-4 a c$ is positive because

$$
b^{2}-4 a c=\operatorname{det}(\Sigma) \operatorname{det}\left(\Sigma^{(12)}\right) .
$$

Since

$$
\operatorname{det}(\Sigma)+(-1)^{m} \cdot 2 \prod_{i=1}^{m} \sigma_{i-1, i}=\operatorname{det}\left(\Sigma^{(12)}\right)-(-1)^{m} \cdot 2 \prod_{i=1}^{m} \sigma_{i-1, i}
$$

we can write

$$
\begin{aligned}
b^{2}= & {\left[\operatorname{det}(\Sigma)+2 \sigma_{12}^{2} \operatorname{det}\left(\Sigma_{[m] \backslash\{1,2\},[m] \backslash\{1,2\}}\right)+(-1)^{m} \cdot 2 \prod_{i=1}^{m} \sigma_{i-1, i}\right] \times } \\
& {\left[\operatorname{det}\left(\Sigma^{(12)}\right)+2 \sigma_{12}^{2} \operatorname{det}\left(\Sigma_{[m] \backslash\{1,2\},[m] \backslash\{1,2\}}\right)-(-1)^{m} \cdot 2 \prod_{i=1}^{m} \sigma_{i-1, i}\right] . }
\end{aligned}
$$

Multiplying out the product, and using (5.7) once more, yields that

$$
\begin{aligned}
& b^{2}=\operatorname{det}(\Sigma) \operatorname{det}\left(\Sigma^{(12)}\right)+4 \sigma_{12}^{4} \operatorname{det}\left(\Sigma_{[m] \backslash\{1,2\},[m] \backslash\{1,2\}}\right)^{2}+4 \prod_{i=1}^{m} \sigma_{i-1, i}^{2}+ \\
& 2 \sigma_{12}^{2} \operatorname{det}\left(\Sigma_{[m] \backslash\{1,2\},[m] \backslash\{1,2\}}\right)\left(\operatorname{det}(\Sigma)+\operatorname{det}\left(\Sigma^{(12)}\right)\right) .
\end{aligned}
$$

Expansion of determinants shows that

$$
\begin{aligned}
\operatorname{det}(\Sigma)=\sigma_{11} \operatorname{det}\left(\Sigma_{[m] \backslash\{1\},[m] \backslash\{1\}}\right)-\sigma_{12}^{2} \operatorname{det}\left(\Sigma_{[m] \backslash\{1,2\},[m] \backslash\{1,2\}}\right) & \\
& -\sigma_{1 m}^{2} \operatorname{det}\left(\Sigma_{[m-1] \backslash\{1\},[m-1] \backslash\{1\}}\right)-(-1)^{m} \cdot 2 \prod_{i=1}^{m} \sigma_{i-1, i} .
\end{aligned}
$$

Hence,

$$
\begin{aligned}
\operatorname{det}(\Sigma)+ & \operatorname{det}\left(\Sigma^{(12)}\right)=2 \sigma_{11} \operatorname{det}\left(\Sigma_{[m] \backslash\{1\},[m] \backslash\{1\}}\right) \\
& -2 \sigma_{12}^{2} \operatorname{det}\left(\Sigma_{[m] \backslash\{1,2\},[m] \backslash\{1,2\}}\right)-2 \sigma_{1 m}^{2} \operatorname{det}\left(\Sigma_{[m-1] \backslash\{1\},[m-1] \backslash\{1\}}\right) .
\end{aligned}
$$

Moreover, we find from another expansion that

$$
\begin{aligned}
& \operatorname{det}\left(\Sigma_{[m] \backslash\{2\},[m] \backslash\{2\}}\right)= \\
& \quad \sigma_{11} \operatorname{det}\left(\Sigma_{[m] \backslash\{1,2\},[m] \backslash\{1,2\}}\right)+\sigma_{1 m}^{2} \operatorname{det}\left(\Sigma_{[m-1] \backslash\{1,2\},[m-1] \backslash\{1,2\}}\right) .
\end{aligned}
$$


Combining (5.8), (5.9) and (5.10), we obtain that our claim (5.6) holds if

$$
\begin{aligned}
& \sigma_{12}^{2} \sigma_{1 m}^{2} \operatorname{det}\left(\Sigma_{[m] \backslash\{1\},[m] \backslash\{1\}}\right) \operatorname{det}\left(\Sigma_{[m-1] \backslash\{1,2\},[m-1] \backslash\{1,2\}}\right)= \\
& \quad \sigma_{12}^{2} \sigma_{1 m}^{2} \operatorname{det}\left(\Sigma_{[m] \backslash\{1,2\},[m] \backslash\{1,2\}}\right) \operatorname{det}\left(\Sigma_{[m-1] \backslash\{1\},[m-1] \backslash\{1\}}\right)-\prod_{i=1}^{m} \sigma_{i-1, i}^{2} .
\end{aligned}
$$

However, all determinants appearing in (5.11) are determinants of tridiagonal matrices and thus (5.11) can be shown to hold by an induction on $m$.

As just established, $b^{2}-4 a c>0$. Moreover, since we assume that both $\operatorname{det}(\Sigma)$ and $\operatorname{det}\left(\Sigma^{(12)}\right)$ are positive it holds that $b>0$; see e.g. (5.8). In addition, $a$ and $c$ are both negative, and it follows from the usual formula for the solutions of a quadratic equation that all four solutions to the quartic equation in (5.5) are real. Hence, $\gamma_{12}$ is real and, by symmetry, the same is true for all other components of a solution to (5.3a) $-5.3 \mathrm{~b}$.

In the final step that completes the proof of sufficiency of the condition in Theorem 5.3. we show that for a generic choice of $\Sigma$, the equations (5.3a)- $5.3 \mathrm{~b}$ ) indeed have at least one complex solution. We are able to restrict attention to generic choices of the entries of $\Sigma$ because $\operatorname{im}\left(\phi_{C_{m}}\right)$ is closed and full-dimensional in $\mathbb{S}_{\succeq 0}\left(C_{m}\right)$. In particular, we may assume that $\sigma_{i-1, i} \neq 0$ for all $i \in[m]$. This implies that a solution of (5.3a $-(5.3 \mathrm{~b})$ has all components non-zero. Moreover, the solution set of (5.3a)-(5.3b) is identical, up to sign, to that of the rational system

$$
\gamma_{i, i+1}^{2}+\frac{\sigma_{i-1, i}^{2}}{\gamma_{i-1, i}^{2}}=\sigma_{i i}, \quad i=1, \ldots, m .
$$

Or simpler yet, setting $x_{i}=\gamma_{i-1, i}$ (in particular, $x_{0} \equiv x_{m}$ ), the solution set corresponds exactly to the solution set of the polynomial system

$$
x_{i+1}^{2} x_{i}^{2}-\sigma_{i i} x_{i}^{2}+\sigma_{i-1, i}^{2}=0, \quad i=1, \ldots, m,
$$

in the torus $\left(\mathbb{C}^{*}\right)^{m}=(\mathbb{C} \backslash\{0\})^{m}$.

Lemma 5.6. For generic choices of the coefficients $\sigma_{i j}$, the equations (5.12) have $2^{m+1}$ solutions in the torus $\left(\mathbb{C}^{*}\right)^{m}$.

Proof. We can rewrite the equation system in (5.12) as

$$
x_{i+1}^{2}=\sigma_{i i}-\frac{\sigma_{i-1, i}^{2}}{x_{i}^{2}}, \quad i=1, \ldots, m .
$$

Solving the equation for $i=1$ for $x_{2}$, plugging the result into the equation for $i=2$ and solving for $x_{3}$, and continuing on in this fashion with all of the first $m-1$ equations, we can write for each $i=2, \ldots, m$,

$$
x_{i}^{2}=\frac{a_{i} x_{1}^{2}+b_{i}}{c_{i} x_{1}^{2}+d_{i}}
$$

where $a_{i}, b_{i}, c_{i}, d_{i}$ are polynomials in the coefficients $\sigma_{k k}, \sigma_{k-1, k}^{2}$. From the last equation, we then obtain that

$$
x_{1}^{2}=\frac{a x_{1}^{2}+b}{c x_{1}^{2}+d},
$$

where $a, b, c, d$ are again polynomials in the coefficients $\sigma_{k k}, \sigma_{k-1, k}^{2}$. 
Now specialize to the case of all $\sigma_{k k}=1$ and all $\sigma_{k-1, k}^{2}=-1$. Then we get

$$
x_{2}^{2}=\frac{x_{1}^{2}+1}{x_{1}^{2}}, \quad x_{3}^{2}=\frac{2 x_{1}^{2}+1}{x_{1}^{2}+1}, \ldots,
$$

and finally equation (5.13) becomes

$$
x_{1}^{2}=\frac{F_{m+1} x_{1}^{2}+F_{m}}{F_{m} x_{1}^{2}+F_{m-1}},
$$

where $F_{m}$ is the $m^{\text {th }}$ term of the Fibonacci sequence $1,1,2,3, \ldots$ Clearing denominators, (5.14) simplifies to $x_{1}^{4}-x_{1}^{2}-1=0$. This equation is obviously not identical to $x^{4}=0$ or $x^{2}=0$, and its discriminant is not identically zero. The coefficients in (5.13) being polynomial, it follows that for generic complex numbers $\sigma_{k k}, \sigma_{k-1, k}^{2}$, the equation in (5.13) has two distinct non-zero solutions up to sign, and the system (5.12) has $2^{m+1}$ solutions.

Lemma 5.7. For any positive definite matrix $\Sigma=\left(\sigma_{i j}\right)$ in image of $\phi_{C_{m}}$, the fiber $\left\{\gamma: \phi_{C_{m}}(\gamma)=\Sigma\right.$ and $\gamma_{i,\{i\}}=0$ for all $\left.i=1, \ldots, m\right\}$ consists of exactly $2^{m+1}$ elements, or two elements up to sign.

Proof. First consider the case when all $\sigma_{i, i+1}$ are non-zero. Since the elements of the fiber are in bijection with the solutions of (5.12) under setting $x_{i}=\gamma_{i-1, i}$, it suffices to show that (5.12) has $2^{m+1}$ solutions. For generic $\Sigma$, this was done in Lemma 5.6. For an arbitrary $\Sigma$, let $\Sigma_{n}$ be a sequence of generic matrices in $\operatorname{im}\left(\phi_{C_{m}}\right)$ that converges to $\Sigma$. Each of the $2^{m+1}$ solutions for $\Sigma_{n}$ can be expressed in terms of radicals using (5.5) and (5.12), and each of these $2^{m+1}$ sequences converge to solutions of (5.12) for $\Sigma$ by continuity. Moreover the limit points are distinct because the discriminant $b^{2}-4 a c$ in (5.5) is positive for $\Sigma$ as shown the proof of Lemma 5.5 and all $x_{i}$ in a solution must be non-zero.

Now suppose $\sigma_{12}=0$. In this case Lemma 5.5 still holds but Lemma 5.6] does not apply. We will now show that the system (5.3a)-(5.3b) still has $2^{m+1}$ complex solutions. Since $\sigma_{12}=0$, we have either $\gamma_{12}=0$ or $\gamma_{21}=0$. If $\gamma_{21}=0$, then we can determine $\gamma_{23}, \gamma_{32}, \gamma_{34}, \gamma_{43}, \ldots, \gamma_{1, m}, \gamma_{12}$ in that order along the cycle, using (5.3a) and (5.3b) alternatingly. These equations show that the sequence of $\gamma_{i j}$ obtained this way is unique up to sign unless we get $\gamma_{i, i+1}=0$ for some $i$. However, if $\gamma_{i, i+1}=0$, then the principal submatrix of $\Sigma$ indexed by $\{2,3, \ldots, i\}$ would be equal to $\Gamma(\gamma) \Gamma(\gamma)^{T}$ where $\Gamma(\gamma)$ is defined as in the introduction for the subgraph on vertices $2,3, \ldots, i$ and edges $\{2,3\}, \ldots,\{i-1, i\}$. Then $\Gamma(\gamma)$ would have more rows than non-zero columns since $\gamma_{i,\{i\}}=0$ for all $i$, so $\Gamma(\gamma) \Gamma(\gamma)^{T}$ would not have full rank, contradicting the hypothesis that $\Sigma$ is positive definite. Hence $\gamma_{i, i+1} \neq 0$ for all $i$, and setting $\gamma_{21}=0$ determines all other $\gamma_{i j}$ up to sign. There are two choices of signs for each pair $\gamma_{i, i+1}$ and $\gamma_{i+1, i}$, even if $\sigma_{i, i+1}=0$, so there are $2^{m}$ solutions with $\gamma_{21}=0$.

By symmetry, if $\gamma_{12}=0$, then we can determine $\gamma_{1, m}, \gamma_{m, 1}, \gamma_{m, m-1}, \ldots, \gamma_{23}, \gamma_{21}$ in that order (going around the cycle in the other direction, with $\gamma_{i, i-1} \neq 0$ in this case), so there are $2^{m}$ solutions when $\gamma_{12}=0$ also. We cannot have both $\gamma_{21}=0$ and $\gamma_{12}=0$ because that would imply that $\Gamma(\gamma) \Gamma(\gamma)^{T}$ is singular. So there are $2^{m+1}$ distinct solutions (two solutions up to sign) to the system (5.3a)-(5.3b) when $\sigma_{12}=0$. By symmetry, there are $2^{m+1}$ solutions for every $\Sigma \in \operatorname{im}\left(\phi_{C_{m}}\right)$. 


\section{Statistical MODELS AND BIPARTIte ACYCLIC DigRAPHS}

In probability theory, positive semidefinite matrices arise as covariance matrices of random vectors. When the random vector $Y=\left(Y_{1}, \ldots, Y_{m}\right)$ is Gaussian (has a multivariate normal distribution) with covariance matrix $\Sigma=\left(\sigma_{i j}\right)$, then $\sigma_{i j}=0$ is equivalent to the stochastic independence of the two random variables $Y_{i}$ and $Y_{j}$. Hence, the convex cone $\mathbb{S}_{\succeq 0}^{m}(G)$ of positive semidefinite matrices with zeros at the non-edges of a graph $G$ collects all covariance matrices for which the components of $Y$ exhibit a pattern of independences.

For a simplicial complex $\Delta$ on $[m]$ with underlying graph $G$, the map $\phi_{\Delta}$ traces out a full-dimensional subset of $\mathbb{S}_{\succeq 0}^{m}(G)$. This subset arises quite naturally for random vectors whose components are linear combinations of a set of independent random variables. We review this construction next.

Let $\Delta_{2}$ be the set of all faces in $\Delta$ that have cardinality at least two. Introduce the random variables $\varepsilon_{i}, i \in[m]$, and $H_{F}, F \in \Delta_{2}$. Suppose the random variables $H_{F}$ are mutually independent with a standard normal distribution, denoted $\mathcal{N}(0,1)$. Suppose further that the $\varepsilon_{i}$ are mutually independent, independent of the $H_{F}$, and distributed as $\varepsilon_{i} \sim \mathcal{N}\left(0, \gamma_{i,\{i\}}^{2}\right)$ where $\gamma_{i,\{i\}}^{2}$ is the variance. Define new random variables $Y_{1}, \ldots, Y_{m}$ as linear combinations:

$$
Y_{i}=\sum_{F \in \Delta_{2}: i \in F} \gamma_{i, F} H_{F}+\varepsilon_{i}, \quad i \in[m] .
$$

Proposition 6.1. The random vector $Y=\left(Y_{1}, \ldots, Y_{m}\right)$ defined by 6.1) has the positive semidefinite matrix $\phi_{\Delta}(\gamma)$ as covariance matrix.

Proof. Write $I$ for the identity matrix (of the appropriate size). Let $\varepsilon=\left(\varepsilon_{1}, \ldots, \varepsilon_{m}\right)$ and $H=\left(H_{F}: F \in \Delta_{2}\right)$. The concatenation $(\varepsilon, H)$ is a random vector with the diagonal $|\Delta| \times|\Delta|$ covariance matrix

$$
\Omega=\left(\begin{array}{cc}
\operatorname{diag}\left(\gamma_{i,\{i\}}^{2}\right) & 0 \\
0 & I
\end{array}\right)
$$

Let $\Gamma_{2}(\gamma)$ be the submatrix of $\Gamma(\gamma)$ obtained by retaining only the columns corresponding to faces in $\Delta_{2}$; recall (1.1). Define the $|\Delta| \times|\Delta|$ matrix

$$
\Lambda=\left(\begin{array}{cc}
I & -\Gamma_{2}(\gamma) \\
0 & I
\end{array}\right) \text {. }
$$

Multiplying $\Lambda$ with $(Y, H)^{T}$ gives the vector $(\varepsilon, H)^{T}$. By standard results about linear combinations of random variables, it follows that the Gaussian random vector $(Y, H)$ has covariance matrix $\Lambda^{-1} \Omega \Lambda^{-T}$. The covariance matrix of $Y$ alone is the principal submatrix given by the first $m$ rows and columns of $\Lambda^{-1} \Omega \Lambda^{-T}$. The inverse $\Lambda^{-1}$ is obtained by negating the upper right block, which becomes simply $\Gamma_{2}(\gamma)$. It follows that, as claimed,

$$
\operatorname{diag}\left(\gamma_{i,\{i\}}^{2}\right)+\Gamma_{2}(\gamma) \Gamma_{2}(\gamma)^{T}=\Gamma(\gamma) \Gamma(\gamma)^{T}=\phi_{\Delta}(\gamma) .
$$

In the field of graphical statistical modelling, it is customary to visualize an equation system such as (6.1) by means of an acyclic digraph; see for instance [DSS09, Chap. 3]. Here, we draw the digraph $D_{\Delta}$ that has vertex set $\Delta$ and the edges $F \rightarrow\{i\}$ for all pairs of an index $i \in[m]$ and a face $F \in \Delta_{2}$ with $i \in F$. Note that $D_{\Delta}$ is bipartite with respect to the partitioning $\Delta=\Delta_{1} \cup \Delta_{2}$, where 

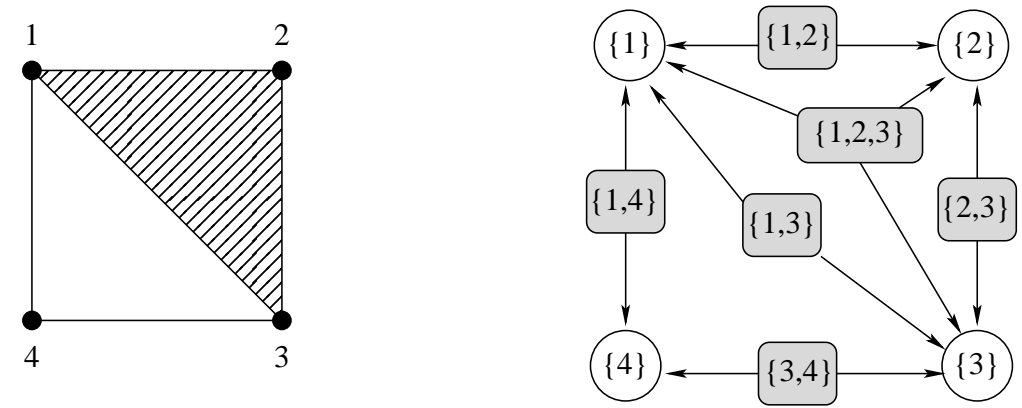

Figure 1. A simplicial complex (left) and the acyclic bipartite digraph corresponding to it (right).

$\Delta_{1}=\{\{i\}: i \in[m]\}$ are the singleton faces and $\Delta_{2}$ was defined above. See Figure 1 for an example.

In the setup of Proposition 6.1 the random variables $Y_{i}$ are functions of the hidden variables $H_{F}$, up to the noise given by the $\varepsilon_{i}$. There is a dual construction in which the hidden variables are functions of the observed variables. Suppose that the random variables $\bar{Y}_{i}$ are mutually independent and normally distributed as $\bar{Y}_{i} \sim \mathcal{N}\left(0,1 / \gamma_{i,\{i\}}^{2}\right)$ with $\gamma_{i,\{i\}}^{2} \neq 0$. Suppose further that $\nu_{F}, F \in \Delta_{2}$, are mutually independent $\mathcal{N}(0,1)$ random variables that are also independent of the $\bar{Y}_{i}$. Define random variables $\bar{H}_{F}$ as linear combinations:

$$
\bar{H}_{F}=\sum_{i \in[m]: i \in F} \gamma_{i, F} \bar{Y}_{i}+\nu_{F}, \quad F \in \Delta_{2} .
$$

Note that this equation system is associated with the bipartite acyclic digraph obtained by reversing the direction of all edges in $D_{\Delta}$.

Proposition 6.2. If the random vector $\bar{H}=\left(\bar{H}_{F}: F \in \Delta_{2}\right)$ is defined by 6.4), then the positive definite matrix $\phi_{\Delta}(\gamma)$ is the inverse of the covariance matrix of the conditional distribution of $\bar{Y}$ given $\bar{H}$.

Proof. Concatenating $\bar{Y}$ and $\bar{H}$ yields a Gaussian random vector with covariance matrix

$$
\Lambda^{-T} \Omega^{-1} \Lambda^{-1}=\left(\begin{array}{cc}
\operatorname{diag}\left(1 / \gamma_{i,\{i\}}^{2}\right) & \operatorname{diag}\left(1 / \gamma_{i,\{i\}}^{2}\right) \Gamma_{2}(\gamma) \\
\Gamma_{2}(\gamma)^{T} \operatorname{diag}\left(1 / \gamma_{i,\{i\}}^{2}\right) & I+\Gamma_{2}(\gamma)^{T} \operatorname{diag}\left(1 / \gamma_{i,\{i\}}^{2}\right) \Gamma_{2}(\gamma)
\end{array}\right),
$$

where we have reused the matrices appearing in (6.2) and (6.3). By standard results about conditional distributions of Gaussian random vectors, the covariance matrix of the conditional distribution of $\bar{Y}$ given $\bar{H}$ is the Schur complement

$$
\begin{aligned}
& \operatorname{diag}\left(\gamma_{i,\{i\}}^{2}\right)^{-1}- \\
& \operatorname{diag}\left(\gamma_{i,\{i\}}^{2}\right)^{-1} \Gamma_{2}(\gamma)\left(I+\Gamma_{2}(\gamma)^{T} \operatorname{diag}\left(\gamma_{i,\{i\}}^{2}\right)^{-1} \Gamma_{2}(\gamma)\right)^{-1} \Gamma_{2}(\gamma)^{T} \operatorname{diag}\left(\gamma_{i,\{i\}}^{2}\right)^{-1} .
\end{aligned}
$$

It follows from the matrix inversion lemma that the inverse of the conditional covariance matrix is

$$
\operatorname{diag}\left(\gamma_{i,\{i\}}^{2}\right)+\Gamma_{2}(\gamma) \Gamma_{2}(\gamma)^{T}=\phi_{\Delta}(\gamma)
$$


According to Proposition 6.2 positive definite matrices in $\operatorname{im}\left(\phi_{\Delta}\right)$ also arise as inverses of conditional covariance matrices. Zeros in the inverse of the covariance matrix of a Gaussian random vector have an appealing interpretation in terms of conditional independence; see again [DSS09, Chap. 3].

\section{ACKNOWLEDGMENTS}

We thank Anton Leykin, Sonja Petrovic, Bernd Sturmfels, and Caroline Uhler for helpful discussions and anonymous referees for detailed comments and for suggesting a simpler alternative proof of Lemma 5.6, which we had previously proven by applying Bernstein's theorem. Josephine Yu was supported by an NSF postdoctoral research fellowship. Mathias Drton was supported by the NSF under Grant No. DMS-0746265 and by an Alfred P. Sloan Fellowship.

\section{REFERENCES}

[AHMR88] Jim Agler, J. William Helton, Scott McCullough, and Leiba Rodman, Positive semidefinite matrices with a given sparsity pattern, Proceedings of the Victoria Conference on Combinatorial Matrix Analysis (Victoria, BC, 1987), vol. 107, 1988, pp. 101-149. MR960140 (90h:15030)

[Bar08] David Barber, Clique matrices for statistical graph decomposition and parameterising restricted positive definite matrices, Proceedings of the 24th Conference in Uncertainty in Artificial Intelligence (David A. McAllester and Petri Myllymäki, eds.), AUAI Press, 2008, pp. 26-33.

[CDS95] Dragoš M. Cvetković, Michael Doob, and Horst Sachs, Spectra of graphs, third ed., Johann Ambrosius Barth, Heidelberg, 1995, Theory and applications. MR1324340 (96b:05108)

[CW96] D. R. Cox and Nanny Wermuth, Multivariate dependencies, Monographs on Statistics and Applied Probability, vol. 67, Chapman \& Hall, London, 1996, Models, analysis and interpretation. MR1456990 (98m:62003)

[DP07] Mathias Drton and Michael D. Perlman, Multiple testing and error control in Gaussian graphical model selection, Statist. Sci. 22 (2007), no. 3, 430-449. MR2416818

[DSS09] Mathias Drton, Bernd Sturmfels, and Seth Sullivant, Lectures on algebraic statistics, Birkhäuser Verlag, Basel, Switzerland, 2009.

[HPR89] J. W. Helton, S. Pierce, and L. Rodman, The ranks of extremal positive semidefinite matrices with given sparsity pattern, SIAM J. Matrix Anal. Appl. 10 (1989), no. 3, 407-423. MR1003106 (90j:05094)

[Lau01] Monique Laurent, On the sparsity order of a graph and its deficiency in chordality, Combinatorica 21 (2001), no. 4, 543-570. MR1863577 (2002i:05080)

[PDB07] Jesus Palomo, David B. Dunson, and Ken Bollen, Bayesian structural equation modeling, Handbook of Latent Variable and Related Models (Sik-Yum Lee, ed.), Elsevier, Amsterdam, 2007, pp. 163-188.

[PPS89] Vern I. Paulsen, Stephen C. Power, and Roger R. Smith, Schur products and matrix completions, J. Funct. Anal. 85 (1989), no. 1, 151-178. MR1005860 (90j:46051)

[RS02] Thomas Richardson and Peter Spirtes, Ancestral graph Markov models, Ann. Statist. 30 (2002), no. 4, 962-1030. MR1926166 (2003h:60017)

$\left[\mathrm{SRM}^{+} 98\right]$ Peter Spirtes, Thomas Richardson, Christopher Meek, Richard Scheines, and Clark Glymour, Using path diagrams as a structural equation modelling tool, Sociological Methods and Research 27 (1998), 182-225.

[STD10] Seth Sullivant, Kelli Talaska, and Jan Draisma, Trek separation for Gaussian graphical models, Ann. Statist. 38 (2010), no. 3, 1665-1685.

Department of Statistics, The University of Chicago, Chicago, Illinois, U.S.A.

E-mail address: drton@uchicago.edu

School of Mathematics, Georgia Institute of Technology, Atlanta, Georgia, U.S.A.

E-mail address: josephine.yu@math.gatech.edu 